Bull. Korean Math. Soc. 49 (2012), No. 4, pp. 761-765

http://dx.doi.org/10.4134/BKMS.2012.49.4.761

\title{
STRONG COHOMOLOGICAL RIGIDITY OF A PRODUCT OF PROJECTIVE SPACES
}

\author{
Suyoung Choi And Dong Youp Suh
}

\begin{abstract}
We prove that for a toric manifold (respectively, a quasitoric manifold) $M$, any graded ring isomorphism $H^{*}(M) \rightarrow H^{*}\left(\prod_{i=1}^{m} \mathbb{C} P^{n_{i}}\right)$ can be realized by a diffeomorphism (respectively, a homeomorphism) $\prod_{i=1}^{m} \mathbb{C} P^{n_{i}} \rightarrow M$.
\end{abstract}

\section{Introduction}

The cohomological rigidity problem for toric manifolds asks whether the integral cohomology ring of a toric manifold determines its topological type or not. So far, there is no negative answer to the question but some positive results. In [2], the authors with M. Masuda show that if $M$ is a toric manifold whose cohomology ring is isomorphic to that of $\prod_{i=1}^{m} \mathbb{C} P^{n_{i}}$, a product of complex projective spaces, then $M$ is actually diffeomorphic to $\prod_{i=1}^{m} \mathbb{C} P^{n_{i}}$, which gives a positive result to the cohomological rigidity problem.

On the other hand, one might ask a stronger question as follows. Throughout this paper, $H^{*}(X)$ denotes the integral cohomology ring of a topological space $X$.

Problem 1.1. Let $M$ and $N$ be toric manifolds, and $\varphi: H^{*}(N) \rightarrow H^{*}(M)$ a graded ring isomorphism. Then, does there exist a homeomorphism or a diffeomorphism $f: M \rightarrow N$ such that $f^{*}=\varphi$ ?

We call this the strong cohomological rigidity problem for toric manifolds. Problem 1.1 for homeomorphism is still open. However, the answer to this question for diffeomorphism is negative in general; for instance, it is shown

Received March 29, 2011.

2010 Mathematics Subject Classification. Primary 57S25; Secondary 22F30.

Key words and phrases. product of projective spaces, generalized Bott manifold, strong cohomological rigidity, toric manifold, quasitoric manifold.

The first author was supported by the new faculty research fund of Ajou University and Basic Science Research Program through the National Research Foundation of Korea(KRF) funded by the Ministry of Education, Science and Technology (2011-0024975).

The second author is partially supported by Basic Science Research Program through the National Research Foundation of Korea(NRF) funded by the Ministry of Education, Science and Technology (2011-0001181). 
by Friedman and Morgan [5] that some cohomology ring automorphism of $\mathbb{C} P^{2} \# 10 \overline{\mathbb{C} P^{2}}$ is not induced by its self-diffeomorphism while it is a toric manifold. Nevertheless, we do conjecture that the strong cohomological rigidity (even for diffeomorphism) holds for some specific subclass of toric manifolds such as generalized Bott manifolds [3, Section 5].

A generalized Bott tower of height $m$ is a sequence of $\mathbb{C} P^{n_{i}}$-bundles with $n_{i} \geq 1$ :

$$
B_{n} \stackrel{\pi_{n}}{\longrightarrow} B_{n-1} \stackrel{\pi_{n-1}}{\longrightarrow} \cdots \stackrel{\pi_{2}}{\longrightarrow} B_{1} \stackrel{\pi_{1}}{\longrightarrow} B_{0}=\{\text { a point }\}
$$

where each $\pi_{i}: B_{i} \rightarrow B_{i-1}$ for $i=1, \ldots, m$ is the projectivization of a Whitney sum of $n_{i}+1$ complex line bundles over $B_{i-1}$. We call $B_{i}$ an $i$-stage generalized Bott manifold or a generalized Bott manifold of height $i$. If all fibration in (1) are trivial, $B_{m}$ is $\prod_{i=1}^{m} \mathbb{C} P^{n_{i}}$.

In this article, we would like to answer to Problem 1.1 for the case when $N=\prod_{i=1}^{m} \mathbb{C} P^{n_{i}}$. Namely, we prove the following theorem.

Theorem 1.2. Let $M$ be a toric manifold. If there is a graded ring isomorphism $\varphi: H^{*}(M) \rightarrow H^{*}\left(\prod_{i=1}^{m} \mathbb{C} P^{n_{i}}\right)$, then there is a diffeomorphism $f: \prod_{i=1}^{m} \mathbb{C} P^{n_{i}} \rightarrow M$ such that $f^{*}=\varphi$.

Combining Theorem 1.2 with [1, Theorem 8.1], we obtain the following corollary which generalizes $\left[6\right.$, Theorem 5.1] treating the case where $n_{i}=1$ for any $i$. Note that a quasitoric manifold is a topological analogue of toric manifold, which is introduced by Davis and Januszkiewicz in [4].

Corollary 1.3. Let $M$ be a quasitoric manifold. If there is a graded ring isomorphism $\varphi: H^{*}(M) \rightarrow H^{*}\left(\prod_{i=1}^{m} \mathbb{C} P^{n_{i}}\right)$, then there is a homeomorphism $f: \prod_{i=1}^{m} \mathbb{C} P^{n_{i}} \rightarrow M$ such that $f^{*}=\varphi$.

\section{Proof of Theorem 1.2}

Let $R=\mathbb{Z}\left[x_{1}, \ldots, x_{m}\right] /\left\langle x_{i}^{n_{i}+1}: i=1, \ldots, m\right\rangle \cong H^{*}\left(\prod_{i=1}^{m} \mathbb{C} P^{n_{i}}\right)$.

Lemma 2.1. Let $y=\sum_{j=1}^{m} a_{j} x_{j} \in R$ such that $a_{i} \neq 0$ for some $i$. Then $y^{n_{i}} \neq 0$ in $R$.

Proof. Suppose $y^{n_{i}}=0$ on the contrary. Then $y^{n_{i}}$ must lie in the ideal generated by the polynomials $x_{j}^{n_{j}+1}$ for $j=1, \ldots, n$. Since $a_{i} \neq 0, y^{n_{i}}=$ $\left(\sum_{j=1}^{m} a_{j} x_{j}\right)^{n_{i}}$ must contain the nonzero monomial term of $x_{i}^{n_{i}}$. However if a nonzero multiple of a power of $x_{i}$ appear in the ideal generated by $x_{j}^{n_{j}+1}$ for $j=1, \ldots, m$, then the exponent must be at least $n_{i}+1$, which is a contradiction.

Lemma 2.2. If $\psi$ is a graded ring automorphism on $R$, then there exists a permutation $\sigma$ on $\{1, \ldots, m\}$ such that $n_{i}=n_{\sigma(i)}$ for all $i=1, \ldots, m$ and $\psi\left(x_{i}\right)= \pm x_{\sigma(i)}$. 
Proof. Let $\psi\left(x_{i}\right)=\sum_{j=1}^{m} b_{i j} x_{j}$ for $i=1, \ldots, m$. Since $\psi$ is an automorphism, $\operatorname{det} B= \pm 1$, where $B=\left(b_{i j}\right)$. Note that the positive integers $n_{1}, \ldots, n_{m}$ need not be distinct. Let $S=\left\{N_{1}, \ldots, N_{k} \mid N_{1}>\cdots>N_{k}\right\}$ be the set of distinct numbers from $n_{1}, \ldots, n_{m}$, and let $\mu:\{1, \ldots, m\} \rightarrow S$ be the function defined by $\mu(i)=n_{i}$. Let $J_{\ell}=\mu^{-1}\left(N_{\ell}\right)$ for $\ell=1, \ldots, k$.

Claim: $B$ is conjugate to a block upper triangular matrix by a permutation matrix.

Since $x_{i}^{n_{i}+1}=0$ in $R, 0=\psi\left(x_{i}^{n_{i}+1}\right)=\left(\sum_{j=1}^{m} b_{i j} x_{j}\right)^{n_{i}+1}$. Therefore, by Lemma 2.1, $b_{i j}=0$ if $n_{i}<n_{j}$. Hence by an appropriate permutation of the index set $\{1, \ldots, m\}$, we may assume that $n_{1} \geq n_{2} \geq \cdots \geq n_{m}$ and $B$ is an upper triangular matrix of the form

$$
\left(\begin{array}{cccc}
C_{J_{1}} & & & * \\
& C_{J_{2}} & & \\
& & \ddots & \\
0 & & & C_{J_{k}}
\end{array}\right),
$$

where $C_{J_{\ell}}$ is the matrix formed from $b_{i j}$ with $i, j \in J_{\ell}$. This proves the claim.

Now let $J_{<\ell}=\bigcup_{\left\{N \in S \mid N<N_{\ell}\right\}} \mu^{-1}(N)$. By the previous claim, if $k \in J_{\ell}$, then we may write $\psi\left(x_{k}\right)=\sum_{j \in J_{\ell}} b_{k j} x_{j}+\sum_{j \in J_{<\ell}} b_{k j} x_{j}$. Let us denote $z_{\ell}=$ $\sum_{j \in J_{\ell}} b_{k j} x_{j}$ and $w_{\ell}=\sum_{j \in J_{<\ell}} b_{k j} x_{j}$ for simplicity. Then $\psi\left(x_{k}\right)=z_{\ell}+w_{\ell}$. Therefore,

$$
0=\psi\left(x_{k}^{N_{\ell}+1}\right)=z_{\ell}^{N_{\ell}+1}+\left(\begin{array}{c}
N_{\ell}+1 \\
1
\end{array}\right) w_{\ell} z_{\ell}^{N_{\ell}}+\left(\begin{array}{c}
N_{\ell}+1 \\
2
\end{array}\right) w_{\ell}^{2} z_{\ell}^{N_{\ell}-1}+\cdots
$$

We note that $z_{\ell} \neq 0$ since $\operatorname{det} B= \pm 1$. On the other hand, there is no way to get the polynomial equation

$$
-\left(\begin{array}{c}
N_{\ell}+1 \\
1
\end{array}\right) w_{\ell} z_{\ell}^{N_{\ell}}=z_{\ell}^{N_{\ell}+1}+\left(\begin{array}{c}
N_{\ell}+1 \\
2
\end{array}\right) w_{\ell}^{2} z_{\ell}^{N_{\ell}-1}+\cdots
$$

in the ring $R$ unless $w_{\ell}=0$. Hence, $z_{\ell}^{N_{\ell}+1}=0$. But then there is a unique nonzero $b_{i j}$ for $i \in J_{\ell}$, and, hence, $b_{i j}= \pm 1$.

Therefore, we have shown that $B$ is conjugate to a diagonal matrix all of whose diagonal entries are \pm 1 . Therefore if $k \in J_{\ell}$, then $\psi$ sends $x_{k}$ to $\pm x_{i}$ for some $i \in J_{\ell}$.

Corollary 2.3. Any graded ring automorphism $\psi$ on $H^{*}\left(\prod_{i=1}^{m} \mathbb{C} P^{n_{i}}\right)$ is induced by a self-diffeomorphism $g$ on $\prod_{i=1}^{m} \mathbb{C} P^{n_{i}}$, i.e., $g^{*}=\psi$.

Proof. Note that any automorphism $\psi$ on

$$
H^{*}\left(\prod_{i=1}^{m} \mathbb{C} P^{n_{i}}\right)=\mathbb{Z}\left[x_{1}, \ldots, x_{m}\right] /\left\langle x_{i}^{n_{i}+1}: i=1, \ldots, m\right\rangle
$$


of the form $\psi\left(x_{i}\right)= \pm x_{\sigma(i)}$ for some permutation $\sigma$ satisfying $n_{i}=n_{\sigma(i)}$ for all $i$ is realized by composition of permutating factors and reversing orientations of factors of $\prod_{i=1}^{m} \mathbb{C} P^{n_{i}}$, appropriately; namely, $\psi$ is induced by a selfdiffeomorphism on $\prod_{i=1}^{\bar{m}} \mathbb{C} P^{n_{i}}$. Hence the corollary follows from Lemma 2.2.

We are now ready to prove Theorem 1.2. Let $\varphi: H^{*}(M) \rightarrow H^{*}\left(\prod_{i=1}^{m} \mathbb{C} P^{n_{i}}\right)$ be a graded ring isomorphism. As mentioned in the introduction, by $[2$, Theorem 1.1], there is a diffeomorphism $h: M \rightarrow \prod_{i=1}^{m} \mathbb{C} P^{n_{i}}$. Then we have

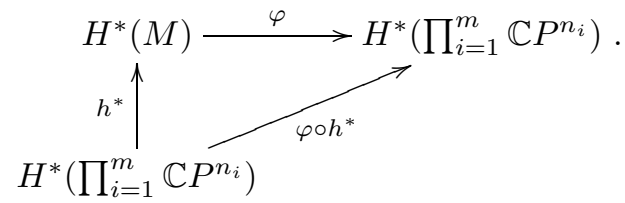

By Corollary 2.3, there is a diffeomorphism

$$
g: \prod_{i=1}^{m} \mathbb{C} P^{n_{i}} \rightarrow \prod_{i=1}^{m} \mathbb{C} P^{n_{i}}
$$

such that $g^{*}=\varphi \circ h^{*}$. Then $\varphi=\left(\varphi \circ h^{*}\right) \circ\left(h^{*}\right)^{-1}=g^{*} \circ\left(h^{-1}\right)^{*}=\left(h^{-1} \circ g\right)^{*}$. Therefore, the diffeomorphism

$$
f:=h^{-1} \circ g: \prod_{i=1}^{m} \mathbb{C} P^{n_{i}} \rightarrow M
$$

induces $\varphi$, which proves the theorem.

\section{References}

[1] S. Choi, M. Masuda, and D. Y. Suh, Quasitoric manifolds over a product of simplices, Osaka J. Math. 47 (2010), no. 1, 1-21.

[2] - Topological classification of generalized Bott manifolds, Trans. Amer. Math. Soc. 362 (2010), no. 2, 1097-1112.

[3] _ Rigidity problems in toric topology, a survey, to appear in Proc. Steklov Inst. Math; arXiv:1102.1359.

[4] M. W. Davis and T. Januszkiewicz, Convex polytopes, Coxeter orbifolds and torus actions, Duke Math. J. 62 (1991), no. 2, 417-451.

[5] R. Friedman and J. W. Morgan, On the diffeomorphism types of certain algebraic surfaces. I, J. Differential Geom. 27 (1988), no. 2, 297-369.

[6] M. Masuda and T. E. Panov, Semi-free circle actions, Bott towers, and quasitoric manifolds, Mat. Sb. 199 (2008), no. 8, 95-122.

SUYOUNG CHOI

Department of Mathematics

AJou UnIVERsity

SuwON 443-749, Korea

E-mail address: schoi@ajou.ac.kr 
Dong Youp SuH

Department of Mathematical Sciences

Korea Advanced Institute of Science and Technology

DAEJEON 305-701, Korea

E-mail address: dysuh@math.kaist.ac.kr 